\title{
The Design and Analysis of Electromagnetic Tracking System
}

\author{
Zhanlei Zhang ${ }^{1}$, Guozhong Liu ${ }^{2}$ \\ ${ }^{1}$ School of Optoelectronic Information \& Communication Engineering, Beijing Information Science \& Technology University, Bei- \\ jing, China; ${ }^{2}$ School of Optoelectronic Information \& Communication Engineering, Beijing Information Science \& Technology \\ University, Beijing, China. \\ Email: lei2231@126.com, liuguozhong@bistu.edu.cn
}

Received December $11^{\text {th }}, 2012$; revised January $10^{\text {th }}, 2013$; accepted January $24^{\text {th }}, 2013$

\begin{abstract}
With the development of optics and microelectronics technology, the portable 3D scanner which is regarded as combination of laser technology, computer science and artificial intelligence have been applied widely in the three-dimensional measuring field, the built-in electromagnetic tracking technology has played an important role in the portable scanner. Combined with the coordinate conversion formula and equipment we can get, using experimental method, we designed the built-in electromagnetic tracking system, using experimental tests, our system can work normally and we accomplished the design and analysis work. This paper is the introduction of this part.
\end{abstract}

Keywords: Electromagnetic Tracking System; Stability; Error

\section{Introduction}

In the 21st century, all kinds of new technology emerge. With the development of optics and microelectronics technology, new 3D scanners hold the market, and in this part western country is in the lead, such as in USA, Polhemus had developed the FastCAN Cobra, in Canada, HandyScan had been used widely. But in china, we only in the study stage. So, it is useful for us to design 3D scanners system.

Electromagnetic tracking system derives from the study of the portable laser scanner's position tracking. Through obtaining the targets' real-time 3D coordinate to connect the image and goals. When a receiving sensor moving in space, electromagnetic tracking system can accurately calculate its position and orientation, it can provide dynamic, real-time measuring position and orientation angle [1]. This system mainly be used in computer fields, such as navigation, ballistic missile tracking, biomechanics, building, tracking, robots, biomechanics, education, virtual reality (VR) and so on. All can obtain the objects' real-time position and direction information by electromagnetic tracking system. It always be used in some places where need accurate tracking.

At present, we accomplished the design and make sure it can work in a small range. In our design, the system is potable and it can work through physical. This is our system's advantage.

\section{The Structure of the Tracking System}

In our study, the electromagnetic tracking system can be decomposed into 4 parts: Transmission Circuit (TC), Receiving Circuit (RC), DSP and Personal Computer (PC).

Transmission Circuit (TC) mainly includes Signal Generating and Processing Circuit (SGPC), Analog Switching Circuit (ASC), Signal Amplification Circuit (SAC) and Electromagnetic Emission Coil (EEC). The signal generating and processing circuit used crystal oscillator. It generates $12 \mathrm{MHz}$ signal. Through frequency division and waveform transform, we get the $9.6 \mathrm{KHz}$ sine signal. Analog switching circuit can be used to control the time of signal radiation with DSP. After signal amplification by signal amplification circuit, the signal can be transferred to the electromagnetic emission coil and launched as the form of electromagnetic waves.

The receiving circuit mainly includes Sensing Receiver (SR), Signal Amplification Circuit (SAC), Band Pass Filter (BPF), Amplitude Detection Circuit (ADC). In the design, we select Honeywell's three axis of magnetic resistance sensor as sensing receiver. It is HMC1043, it has the following characteristics: low cost, high sensitivity, minute extension, low noise, high reliability, strong adaptability, easy installation and so on [2]. Amplitude detection circuit can give us the signal amplitude size what we need. After these, the signal will be transmitted to DSP and then do software processing.

TMS320F2812 is known as a rapid processing DSP, it 
can meet the sampling frequency that we need, and using its $\mathrm{A} / \mathrm{D}$ part, the analog signals can be converted into digital signals. Another role is that it can control the time of signal transmission [3].

PC can give us the data by programming.

All of the component can be illustrated in Figure 1.

The electromagnetic emission coil is stereo ferric core winding circle. In the three directions of the cube we bind multilayer coils. Due to geometric construction of the cube, it can accurately guarantee the three coils orthopedic and homocentric [4]. The model is shown in Figure 2.

DSP mainly have following ports to make all parts together, it is shown in Figure 3.

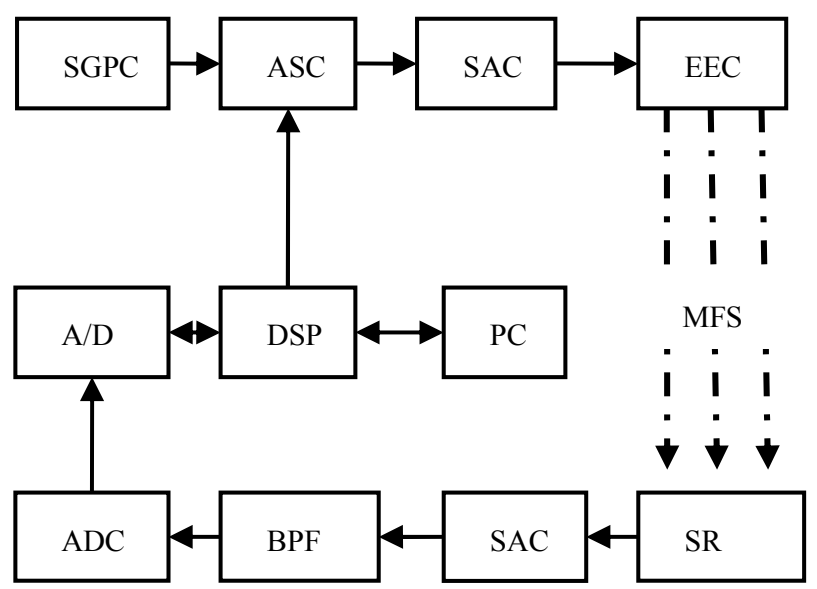

(State: MFS is magnetic field space)

Figure1. The structure of the electromagnetic tracking system.
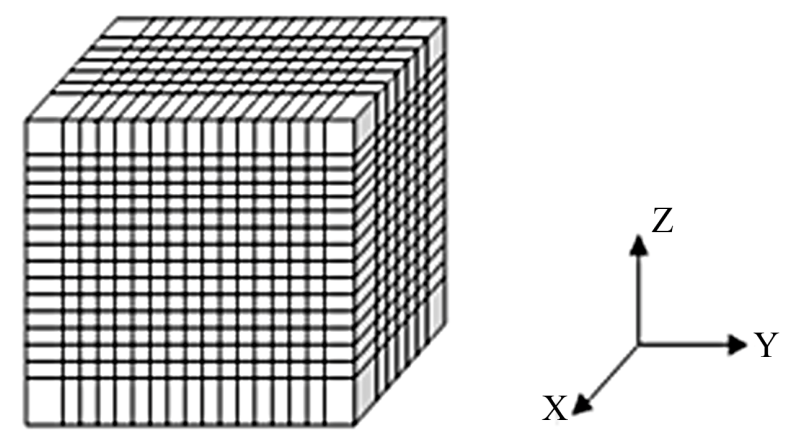

Figure 2. The electromagnetic emission coil.

$$
\begin{gathered}
W=\frac{1}{4} \frac{c^{2}}{\rho^{6}}\left[\begin{array}{ccc}
1+3 \cos ^{2} \alpha \cos ^{2} \beta & 3 \sin \alpha \cos \alpha \cos ^{2} \beta & -\cos \alpha \sin \beta \cos \beta \\
3 \sin \alpha \cos \alpha \cos ^{2} \beta & 1+3 \sin ^{2} \alpha \cos ^{2} \beta & -3 \sin \alpha \sin \beta \cos \beta \\
-3 \cos \alpha \sin \beta \cos \beta & -3 \sin \alpha \sin \beta \cos \beta & 1+3 \sin ^{2} \beta
\end{array}\right] \\
A=\left[\begin{array}{ccc}
\cos \psi \cos \omega & \cos \psi \sin \omega & -\sin \psi \\
\sin \xi \sin \psi \cos \omega-\cos \xi \sin \omega & \sin \psi \sin \xi \sin \omega+\cos \xi \cos \omega & \sin \xi \cos \psi \\
\cos \xi \sin \psi \cos \omega+\sin \xi \sin \omega & \cos \xi \sin \psi \sin \omega+\sin \xi \cos \omega & \cos \xi \cos \psi
\end{array}\right]
\end{gathered}
$$


From (1) we can get:

$$
P(z)=\frac{c^{2}}{\rho^{6}}\left[\sin ^{2} \beta+\frac{1}{4} \cos ^{2} \beta\right]
$$

$P(z)$, in $F$ matrix, is modulus square of received vector of $z$ axis and $G$ is unit matrix.

Using $F$ we can get the calculation formula of $\rho$.

Here we simplify $W$ as:

$$
W=\frac{1}{4} \frac{c^{2}}{\rho^{6}} F
$$

Using (2) we can get the calculation formulas of $\alpha$ and $\beta$ :

$$
\begin{gathered}
\alpha=\arctan \left(F_{23} / F_{13}\right) \\
\beta=\left[\arctan \left(F_{23} / F_{13}\right) / 3\right]^{1 / 2}
\end{gathered}
$$

Using (3) we can get the calculation formulas of $\omega, \xi$ and $\psi$ :

$$
\begin{gathered}
\xi=\arctan \left(A_{23} / A_{33}\right) \\
\omega=\arctan \left(A_{12} / A_{11}\right) \\
\psi=\arctan \left(-A_{13} \cos \omega / A_{11}\right)
\end{gathered}
$$

Based on these formulas we can program for DSP to complete the operation of software.

The relationships between coordinates can be illustrated in Figure 4 [6] and coordinate conversion formula is:

$$
\left[\begin{array}{l}
X^{*} \\
Y^{*} \\
Z^{*}
\end{array}\right]=R\left[\begin{array}{l}
X \\
Y \\
Z
\end{array}\right]+T
$$

Here, $R$ is rotation matrix, it is

$$
R=\left[\begin{array}{lll}
r_{11} & r_{12} & r_{13} \\
r_{21} & r_{22} & r_{23} \\
r_{31} & r_{32} & r_{33}
\end{array}\right]
$$

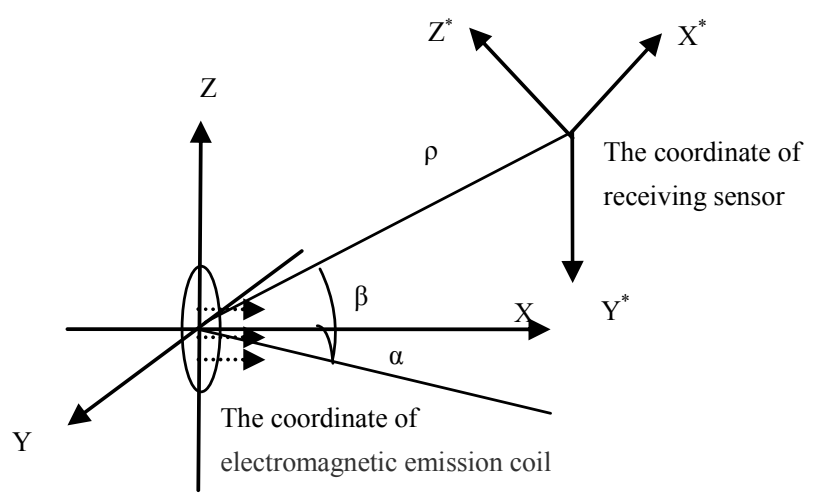

Figure 4. The coordinate relationship.
And $T$ is translation matrix, it is

$$
T=\left[\begin{array}{lll}
t_{x} & t_{y} & t_{z}
\end{array}\right]^{\mathrm{T}}
$$

\section{The Methods of Data Acquisition}

In experiment, by controlling I/O ports, we get the data in sequence.

The principle diagram is expressed in Figure 5.

In practice, by controlling the analog switching, we can implement time-share launch. It can put a measurement cycle divided into three stage, the emission signals can only drive one launch coil at one stage. So, in each stage, only one coil can produce space magnetic field. The HMC1043 get 3 data of one coil in one stage, so we can get 9 data totally in one measurement cycle [7].

\section{Data Collection and Analysis}

\subsection{The Data Collection}

In experiment, the data acquisition part used DSP model of SEED_DPS2812M, it collect analog signals and convert it into digital signals. We can finally get these digital signals in PC.

Here, we just take the collected voltage amplitude for example, not put everything, such as the rotational angles- $\omega, \xi, \psi$ into detail. We collect voltage amplitude of each axis to check our system's stability and errors. In order to find the problem, we collect data several times at one position, each cycle's data as one group. We select three groups of data to state [8], the position is: $\rho=9.50$ $\mathrm{cm}, \alpha=25.00^{\circ} \beta=45.00^{\circ}$, the data are shown in Tables 1-3, and the actual data of amplitude are shown in Table 4.

\subsection{The Analysis of the System}

Analyzing the collected data, we average the three data of every stage of every axis, then calculate the relative error and the relative error are shown in Table 5 .

In reality, we select $\rho$ at the position of $10 \mathrm{~cm}$. Due to larger diameter of the electromagnetic emission coil, it about $5 \mathrm{~cm}$, in fact $\rho$ is the space length from the receiver

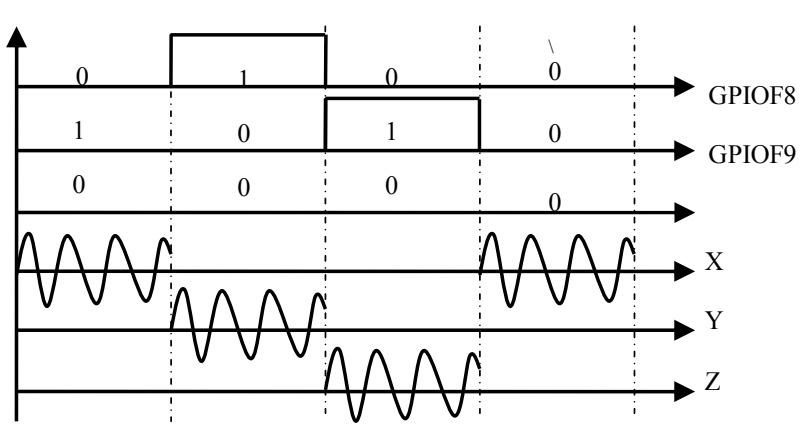

Figure 5. Sequence control schemes. 
Table 1. The first group of amplitude.

\begin{tabular}{cccc}
\hline & $\begin{array}{c}\text { First stage } \\
\text { (collected) }\end{array}$ & $\begin{array}{c}\text { Second stage } \\
\text { (collected) }\end{array}$ & $\begin{array}{c}\text { Third stage } \\
\text { (collected) }\end{array}$ \\
\hline$X$ axi (v) & 0.97 & 0.73 & 0.69 \\
$Y$ axi (v) & 0.92 & 0.69 & 0.08 \\
$Z$ axi (v) & 0.68 & 0.49 & 0.28 \\
\hline
\end{tabular}

Table 2. The second group of amplitude.

\begin{tabular}{lccc}
\hline & $\begin{array}{c}\text { First stage } \\
\text { (collected) }\end{array}$ & $\begin{array}{c}\text { Second stage } \\
\text { (collected) }\end{array}$ & $\begin{array}{c}\text { Third stage } \\
\text { (collected) }\end{array}$ \\
\hline$X$ axis (v) & 0.99 & 0.72 & 0.72 \\
$Y$ axis (v) & 0.99 & 0.73 & 0.08 \\
$Z$ axis (v) & 0.72 & 0.55 & 0.29 \\
\hline
\end{tabular}

Table 3. The third group of amplitude.

\begin{tabular}{lccc}
\hline & $\begin{array}{c}\text { First stage } \\
\text { (collected) }\end{array}$ & $\begin{array}{c}\text { Second stage } \\
\text { (collected) }\end{array}$ & $\begin{array}{c}\text { Third stage } \\
\text { (collected) }\end{array}$ \\
\hline$X$ axis (v) & 1.02 & 0.76 & 0.73 \\
$Y$ axis (v) & 1.04 & 0.74 & 0.11 \\
$Z$ axis (v) & 0.75 & 0.52 & 0.25 \\
\hline
\end{tabular}

Table 4. The actual data of amplitude.

\begin{tabular}{lccc}
\hline & $\begin{array}{c}\text { First stage } \\
\text { (collected) }\end{array}$ & $\begin{array}{c}\text { Second stage } \\
\text { (collected) }\end{array}$ & $\begin{array}{c}\text { Third stage } \\
\text { (collected) }\end{array}$ \\
\hline$X$ axis (v) & 1.00 & 0.75 & 0.70 \\
$Y$ axis (v) & 1.00 & 0.70 & 0.10 \\
$Z$ axis (v) & 0.70 & 0.50 & 0.30 \\
\hline
\end{tabular}

Table 5. The relative error.

\begin{tabular}{cccc}
\hline & First stage & Second stage & Third stage \\
\hline$X$ axis (\%) & 1.00 & 1.78 & 1.90 \\
$Y$ axis (\%) & 1.67 & 2.86 & 10.00 \\
$Z$ axis (\%) & 2.86 & 4.00 & 8.89 \\
\hline
\end{tabular}

center to coil center and the data we get unavoidably exists error. In experiment we find if $\rho$ is bigger than 15 $\mathrm{cm}$ the signal weakening faster. From the table we can find:

1) When the output voltage is small, for example, approach to $0.1 \mathrm{v}$, the relative error is large.

2) Because of time-share launch, in different stage, the output voltage is different, but the voltage of the same axis at different time is basic stable. After many experiments-different position experiments, this can be proved and our system is stable.

3) Due to the differences of position, in the same position for different axis, the error is different; the same axis for different position, the error is also different.

Summing up above and combined with the experiments, the stability of the system is ideal. But due to the limit of the distance between the electromagnetic emission coil and receiver, the error of the system is big and the main factor is the size of the electromagnetic emission coil. In addition, the output voltage amplitude smaller is also one factor.

\section{The Improvement Method and Conclusion}

Now, our system can only work in a small scope, such as the receiver distance is $10 \mathrm{~cm}$ and its precision is only 2 $4 \mathrm{~mm}$, for a well precision, it can reach $0.5 \mathrm{~mm}$, all of this is our system's limitation. Next step we should improve the precision and make it working radius reach 20 $30 \mathrm{~cm}$. In order to improve our system, there are many methods:

1) Improve the electromagnetic emission coil, due to the limit of the distance between the electromagnetic emission coil and the receiver. The coil is smaller the effect will be better. Normally, diameter of the coil should be far less the distance from the coil to receiver. But at the same time, we should ensure the electromagnetic emission coil working at resonance state and transmitted power largest, the circle number of the coil and the area of the coil should meet the need of transmitted power, the coil cannot be made infinitely small.

2) Increase signal magnification of the receiving circuit. In our experiment, in order to reduce the noise interference, the magnification of the signal amplification circuit is just 200 times.

3) Combined with six degree-of-freedom algorithm, do compensation process. In this part we can use Two Interpolation Algorithm to do compensation process for amplitude and angle of output.

At present, we accompliment our design and through the experiment, our system can work stability at a small scope and the precision need improved. In short, we accomplished the design and analysis work and make a good preparation for next section.

\section{REFERENCES}

[1] D. Roetenberg, P. Slycke, A. Ventevogel and P. H. Veltink, "Portable Magnetic Position and Orientation Tracker," Sensors and Actuators, Vol. 135, No. 2, 2007, pp. 426432. doi:10.1016/j.sna.2006.08.020

[2] M. J. Caruso, "Applications of Magnetic Sensors for Low Cost Compass Systems," IEEE Position Locations and Navigation Symposium, San Diego, 13-16 March 2000, pp. 178-184.

[3] K. F. Sun and Q. Lv, "TMS320F2812 Theory and Development," Electronic Industry Press, Beijing, 2005.

[4] H. Hua,Y. Tian and T. Xu, "Dynamic Range Finding Device Using Amplitude-Modulated Continuous Ultrasonic," Journal of Beijing Institute of Technology, Vol. 7, No. 1, 1998, pp. 55-61.

[5] S. L. Sun, D. Y. Yan and C. H. Zhao, "The Research of Six Degree-of-Freedom Electrommagnetic Tracking Sys- 
tem," Electronic Measurement Technology, Vol. 5, No. 1, 2005, pp. 30-31.

[6] T. Xu, Y. T. Wang and D. Y. Yan, "Six Degree of Freedom Electrommagnetic Tracking System for Virtual Reality," Journal Beijing Institute of Technology, Vol. 5, No. 20, 2000, pp. 544-546.
[7] SanHengXing Technology, "TMS320F2812 Theory and Application Example," Electronic Industry Press, Beijing, 2009, pp. 12-14.

[8] R. B. Smith and B. G. Morton, "Elgersma Electronic Compass and Compensation of Large Magnetic Errors for Operation over All Orientations," US Patent 6543146, 2003. 